\title{
Encéphalite auto-immune séronégative
}

\author{
Owais Alsrouji MD, Anza B. Memon MD
}

Citation : CMAJ 2020 November 30;192:E1638. doi : 10.1503/cmaj.200424-f

Voir la version anglaise de l'article ici : www.cmaj.ca/lookup/doi/10.1503/cmaj.200424

$\mathbf{U}$ ne femme de 68 ans ayant des antécédents de polyarthrite rhumatoïde a consulté aux urgences pour un déclin cognitif rapide au cours des 3 mois précédents. L'examen a révélé une récente perte de mémoire à court terme, une faible capacité d'attention, une difficulté à conceptualiser les tâches et une incapacité à exécuter une séquence d'actions. Trois jours après son admission, la patiente a développé une rigidité des membres, une hyperréflexie, un réflexe de sursaut exagéré et une dysautonomie, accompagnées de fluctuations de la tension artérielle et de la fréquence cardiaque.

L'imagerie par résonance magnétique (IRM) du cerveau de la patiente a révélé la présence d'hypersignaux bilatéraux confluents au niveau des noyaux gris centraux (figure 1A). La sérologie auto-immune a confirmé la présence d'un taux d'anticorps antinucléaires de 1:640 (présentation homogène; plage de référence $<1: 80$ ) et un taux élevé d'anticorps antipeptides cycliques citrullinés (> $340 \mathrm{UI} / \mathrm{mL}$ [plage de référence $<7 \mathrm{UI} / \mathrm{mL}]$ ). Concordant avec un diagnostic d'encéphalite auto-immune séronégative, la sérologie s'est révélée négative pour les anticorps antiglutamate décarboxylase, antirécepteur $\mathrm{N}$-méthyl-D-aspartate et antirécepteurs de la glycine. L'analyse du liquide céphalorachidien a montré un taux élevé de protéines, à $203 \mathrm{mg} / \mathrm{dL}$ (plage normale $15-55 \mathrm{mg} / \mathrm{dL}$ ). Les résultats d'autres tests (acides gras à longue chaîne, toxicité, infectiologie et oncologie) se sont révélés négatifs (annexe 1 , accessible en anglais au www.cmaj.ca/lookup/doi/10.1503/ cmaj.200424/tab-related-content). Nous avons diagnostiqué une encéphalite auto-immune.

La patiente a reçu une dose de $1 \mathrm{~g}$ de méthylprednisolone, après quoi un saignement gastro-intestinal s'est déclaré. Deux semaines plus tard, une nouvelle IRM cérébrale a montré une aggravation des anomalies (figure 1B). Cinq cycles de plasmaphérèse ont été réalisés, puis une autre IRM a montré une résolution quasi complète des hypersignaux au niveau des noyaux gris centraux (figure 1C). La patiente a ensuite pris du rituximab, et 6 semaines plus tard, elle présentait un rétablissement neurologique complet.

L'incidence de l'encéphalite auto-immune est de 5 à 10 par 100000 années-personnes ${ }^{1}$. Le diagnostic se fonde sur le tableau clinique d'encéphalomyélite progressive et sur les signes d'encéphalite aux épreuves de neuro-imagerie ${ }^{1,2}$. Les résultats négatifs aux dosages d'anticorps ne permettent pas d'écarter la possibilité d'encéphalite auto-immune, et le risque de morbidité et de mortalité est plus grand chez les patients non traités ${ }^{2}$. Un diagnostic et un traitement rapides, y compris par immunomodulateurs (comme des immunoglobulines par intraveineuse), corticostéroïdes à forte dose et plasmaphérèse, sont associés à de bons résultats. Les patients dont la maladie est agressive ou qui rechutent ont besoin d'un traitement immunosuppresseur d'entretien. 


\section{Références}

1. Graus F, Titulaer MJ, Balu R, et al. A clinical approach to the diagnosis of autoimmune encephalitis. Lancet Neurol 2016;15:391-404.

2. Dutra LA, Abrantes F, Toso FF, et al. Autoimmune encephalitis: a review of diagnosis and treatment. Arq Neuropsiquiatr 2018;76:41-9.

Intérêts concurrents : Aucun déclaré.

Cet article a été révisé par des pairs.

Les auteurs ont obtenu le consentement de la patiente.

Affiliations : Département de neurologie (Alsrouji, Memon), Hôpital Henry Ford; Faculté de médecine (Memon), Université d’État de Wayne, Détroit, Michigan.

Propriété intellectuelle du contenu : Il s'agit d'un article en libre accès distribué conformément aux modalités de la licence Creative Commons Attribution (CC BY-NC-ND 4.0), qui permet l'utilisation, la diffusion et la reproduction dans tout médium à la condition que la publication originale soit adéquatement citée, que l'utilisation se fasse à des fins non commerciales (c.-à-d., recherche ou éducation) et qu'aucune modification ni adaptation n'y soit apportée. Voir : https://creativecommons.org/licenses/by-nc-nd/4.0/deed.fr

Remerciements : Les auteurs remercient Stephanie Stebens pour son aide à la préparation de ce manuscrit.

Correspondance : Anza Memon, amemon2@hfhs.org

Les images cliniques sont choisies pour leur caractère particulièrement intéressant, classique ou impressionnant. Toute soumission d'image de haute résolution claire et bien identifiée doit être accompagnée d'une légende aux fins de publication. On demande aussi une brève explication (300 mots maximum) de la portée éducative des images, et des références minimales. Le consentement écrit du patient au regard de la publication doit être obtenu avant la soumission. 